\title{
A NOVEL RPC BIAS MODEL FOR IMPROVING THE POSITIONING ACCURACY OF SATELLITE IMAGES
}

\author{
Yang Dong, Rong Lei *, Dazhao Fan, Linyu Gu, Song Ji \\ Information Engineering University, No.62 Science Avenue, Zhengzhou, China - (wenku34, leirong, fdzcehui, guly617, \\ jisong_chxy)@163.com
}

Commission II, WG II/1

KEY WORDS: RPC bias model, Positioning accuracy, Block adjustment, Satellite image, TH-1

\begin{abstract}
:
High-precision satellite image geolocation is the basis for advanced processing of satellite image data. Aiming at the optimization of the satellite image positioning accuracy based on rational polynomial coefficients (RPC), we propose an RPC image-space bias model that combines object-space information. Based on a comprehensive analysis of the full-link error of the satellite image geometric imaging process, the real object coordinates are introduced into the RPC correction to make the bias model better fit the actual error. Experiments were performed using several image datasets from the Chinese satellite TianHui-1 (TH-1) and compared with the traditional RPC bias model. The results show that our model has strong robustness and can better correct image positioning errors. Compared with traditional bias models, it can improve the accuracy of plane positioning by approximately 1 pixel.
\end{abstract}

\section{INTRODUCTION}

In the past decade, high-resolution remote sensing satellites have been comprehensively developed and widely used. In all aspects of the intelligent ground observation network providing spatial information services, high-precision satellite image geolocation is the basis for exerting application efficiency and value ( $\mathrm{Li}, 2019)$. However, all sensor models include orbit measurement errors, offset errors caused by attitude measurement errors, and image distortion errors (Cao et al., 2019). Although most of these errors have been calibrated in the laboratory, due to the influence of the thermal and mechanical environment during satellite launch and operation, there is a certain deviation between the laboratory calibration values and the true values, and the direct positioning accuracy of satellite images cannot meet the target requirements. Therefore, it is an important step in satellite image data processing to perform fulllink error analysis and modeling to improve the positioning accuracy.

In the early stage of satellite data processing, the strict geometric imaging model of satellites and high-precision ground control points (GCPs) can generally be used to accurately calibrate the interior and exterior orientation elements of the satellite to improve the direct positioning accuracy of satellite images. The strict geometric model reflects the imaging mechanism of remote sensing satellites. According to the design characteristics of the sensor, using the attitude and orbit measurement equipment carried on the satellite platform, a series of coordinate system transformations can be used to construct a strict geometric model of the satellite. By analyzing the error source of the satellite imaging process, adding error correction parameters to the strict geometric model, and using high-precision ground control points to solve the error correction parameters, the on-orbit geometric calibration of the satellite image can be achieved. A large number of scholars have conducted in-depth research on the design of error correction models and parameter solution methods for satellite on-orbit geometric calibration and have carried out a large number of experiments on satellite images such as WorldView, Pleiades, ZiYuan-3 (ZY-3), and TH-1 (Jiang et al., 2018; Wang et al., 2018).

In the later stages of satellite data processing, controlled or uncontrolled regional bundle adjustment can generally be performed with a satellite rational function model (RFM) to improve the direct positioning accuracy or the consistency of the overall accuracy of satellite images. RFM does not need to consider the imaging information of the sensor, and it is very convenient. RPC parameters have now become the auxiliary parameter of most satellite image products and are distributed to users directly. The on-orbit geometric calibration parameters of satellite images are generally used in the generation of RPC, which has high direct positioning accuracy. However, due to the limitation of the satellite calibration model, as the satellite image moves away from the calibration area or with the passage of time after calibration, the positioning accuracy of the satellite image will gradually deteriorate, and a certain systematic deviation will appear (Yang, 2016; Yang et al., 2017). For advanced product application requirements such as digital elevation models (DEMs) extraction and orthophoto generation, users still need to use control points or constraints to further perform regional bundle adjustment based on the RPC model to meet the accuracy requirements. However, due to the strong correlation between RPC parameters, these parameters cannot be directly solved or optimized as unknowns in regional bundle adjustment. At the same time, these parameters do not have any geometric significance, which brings great difficulties to the geometric interpretation of satellite images and effective compensation of various errors. Regional bundle adjustment of satellite imagery based on an RPC model primarily uses imagespace affine transformation to correct model deviations (Cao et al., 2019; Fraser et al., 2006; Fraser, Hanley 2003, 2005; Grodecki, Dial, 2003; Noh, Howat, 2018; Pan et al., 2018; Tong et al., 2010; Yang et al., 2017; Zhang et al., 2009). However, when the affine transformation model cannot simulate the satellite on-orbit geometric calibration residuals well, the

* Corresponding author 
positioning accuracy of the satellite images will be affected (Cao et al., 2019).

This paper focuses on the optimization of the satellite image positioning accuracy based on an RPC bias model. This paper analyzes the full-link error in the satellite imaging process and proposes a polynomial bias model combining the image space and object space to correct the model deviation. The inconsistent positioning accuracy of the satellite RPC is essentially caused by the instability of the satellite on-orbit geometric calibration model. In the noncalibration area, the thermal and mechanical effects on satellites are inconsistent with the calibration, which results in the instability of the extrapolation of the calibration results. The influence of satellite space thermal characteristics and mechanics are mainly reflected in the attitude measurement and image internal distortion. For high-orbit geostationary satellite images, this situation is more obvious (Dong et al., 2019). These influences show a certain correlation with the satellite's operating position. Therefore, this paper introduces object-space factors and considers image-space correction under object-space conditions to improve the accuracy of the adjustment results based on an RPC model. The remaining chapters of this paper are organized as follows: Section 2 introduces the bias model and method proposed in this paper in detail; Section 3 uses multiple satellite image datasets to experimentally verify the method in this paper and compares it with the traditional bias model; Section 4 summarizes this paper.

\section{METHOD}

This section first analyzes the full-link error in the satellite imaging process and provides a theoretical basis for improving the accuracy of satellite image positioning. Then, it introduces the traditional RPC bias model and analyzes its shortcomings. Finally, the object-related RPC bias model and the specific adjustment process method are given.

\subsection{Full-Link Error Analysis}

According to general satellite image processing, the sources of errors that affect the geometric positioning accuracy of satellite images can be divided into two categories: exterior orientation errors and interior orientation errors (Dong et al., 2019).

The exterior orientation elements are mainly used for the conversion between the camera's coordinate system and the object's coordinate system and describe the actual spatial orientation of the satellite's camera. The exterior orientation elements can be obtained by attitude measurement systems and orbital coordinate measurement systems. However, the limitations of the measurement equipment accuracy and installation technology and changes in the environment will introduce errors into the exterior orientation elements. In general, the exterior orientation error terms mainly include the attitude error, orbit error, camera installation error, and time error. Satellite on-orbit geometric calibration mainly models and calibrates the attitude error, orbit error, and camera installation error. The effect of the time error on the image positioning accuracy is generally negligible. The influence of the attitude error on the geometric positioning accuracy can be divided into three aspects: the rolling angle error causes the imaging light direction to change in the vertical orbit direction, and the image is distorted in the vertical orbit direction; the elevation angle error causes the imaging light direction to change along the orbital direction, causing the image to be distorted along the orbital direction; and the yaw angle error causes the imaging light to rotate as a whole, causing the image to shift in both the orbit and the vertical directions. The influence of the orbital error on the geometric positioning accuracy can also be divided into three aspects: the vertical orbital error, which causes the equivalent translation of the image in the vertical orbital direction; the orbital error, which causes the equivalent translation of the image in the orbital direction; and the radial error, which causes overall image scaling. The camera installation error is the error between the actual camera installation value and the laboratory calibration value. The effect on the geometric positioning accuracy is basically the same as the effect caused by the attitude error. The camera installation error is mainly caused by vibrations of the loading platform during launch and operation and thermal deformations of the loading platform during operation. Satellite loading platforms are affected by solar light of different intensities at different times in different regions, resulting in continuous changes in the heat flow received by each part of the satellite surface. The change in heat flow will cause the deformation of the fixed device inside the satellite to become more negligible, which will affect the relative installation relationship between the camera and the platform (Dong et al., 2019; Wang et al., 2017).

The interior orientation elements describe the conversion relationship between the image's coordinate system and the camera's coordinate system and determine the light vector of the image point in the camera's coordinate system. The interior orientation errors of satellite cameras mainly include the photosensitive surface error, focal length error, and lens distortion error. In the on-orbit geometric calibration of the satellite, the interior orientation error is mainly modeled by a polynomial additional parameter model. The more widely used polynomial additional parameter model is the two-dimensional pointing angle model (Dong et al., 2019; Wang et al., 2017). According to the full-link error analysis, the general satellite onorbit geometric model is:

$$
\left[\begin{array}{c}
\tan \phi_{x} \\
\tan \phi_{y} \\
-1
\end{array}\right]=\lambda^{\prime} R_{\text {body }}^{\text {cam }}(\varphi, \omega, \kappa) R_{\text {orbit }}^{\text {body }} R_{W G S 84}^{\text {orbit }}\left[\left(\begin{array}{c}
X_{g} \\
Y_{g} \\
Z_{g}
\end{array}\right)-\left(\begin{array}{c}
X_{\text {body }} \\
Y_{\text {body }} \\
Z_{\text {body }}
\end{array}\right)\right]
$$

where $\left(\tan \phi_{x}, \tan \phi_{y}\right)$ is the direction of the probe in the camera's coordinate system, $\lambda^{\prime}$ is the scale factor, $(\varphi, \omega, \kappa)$ is the camera installation parameter, $R_{b o d y}^{\text {cam }}$ is the camera installation matrix, $R_{\text {orbit }}^{\text {body }}$ is the transformation matrix for the orbit coordinates to the body coordinates, $R_{W G S 84}^{\text {orbit }}$ is the transformation matrix of the WGS 84 coordinates to the orbit coordinates, $\left[X_{g}, Y_{g}, Z_{g}\right]^{T}$ is the coordinates of the object point under WGS84, and $\left[X_{\text {body }}, Y_{\text {body }}, Z_{\text {body }}\right]^{T}$ is the coordinates of the projection center under WGS84.

Generally, the attitude error and camera installation error are equivalent to the exterior orientation calibration parameter and the low-order terms of the interior orientation calibration parameter, and the orbital error is equivalent to the low-order terms of the interior orientation calibration parameter. The photographic surface error, focal length error, and lens distortion error are equivalent to the interior orientation calibration parameters. After the on-orbit geometric calibration of the satellite, the direct positioning accuracy of the satellite image has been greatly improved. However, for noncalibrated areas, there is still a certain amount of systematic residual error, which can be further improved by the RPC bias model. 


\subsection{Traditional RPC Bias Model}

The mathematical expression of the RFM is a rational polynomial model, and its definition is shown in the following formula.

$$
\begin{aligned}
& \left\{\begin{array}{l}
l=\frac{\operatorname{Num}_{L}(B, L, H)}{\operatorname{Den}_{L}(B, L, H)} l_{s}+l_{0} \\
s=\frac{\operatorname{Num}_{S}(B, L, H)}{\operatorname{Den}_{S}(B, L, H)} s_{s}+s_{0}
\end{array}\right. \\
& \left\{\begin{array}{l}
B=\frac{\phi^{\prime}-\phi_{0}}{\phi_{s}} \\
L=\frac{\lambda^{\prime}-\lambda_{0}}{\lambda_{s}} \\
h=\frac{h^{\prime}-h_{0}}{h_{s}}
\end{array}\right.
\end{aligned}
$$

$$
\left\{\begin{array}{l}
\operatorname{Num}_{L}(B, L, H)=a_{1}+a_{2} \cdot L+a_{3} \cdot B+a_{4} \cdot H+a_{5} \cdot L \cdot B+a_{6} \cdot L \cdot H+ \\
a_{7} \cdot B \cdot H+a_{8} \cdot L^{2}+a_{9} \cdot B^{2}+a_{10} \cdot H^{2}+a_{11} \cdot B \cdot L \cdot H+a_{12} \cdot L^{3}+ \\
a_{13} \cdot L \cdot B^{2}+a_{14} \cdot L \cdot H^{2}+a_{15} \cdot L^{2} \cdot B+a_{16} \cdot B^{3}+a_{17} \cdot B \cdot H^{2}+ \\
a_{18} \cdot L^{2} \cdot H+a_{19} \cdot B^{2} \cdot H+a_{20} \cdot H^{3} \\
\operatorname{Den}_{L}(B, L, H)=b_{1}+b_{2} \cdot L+b_{3} \cdot B+b_{4} \cdot H+b_{5} \cdot L \cdot B+b_{6} \cdot L \cdot H+ \\
b_{7} \cdot B \cdot H+b_{8} \cdot L^{2}+b_{9} \cdot B^{2}+b_{10} \cdot H^{2}+b_{11} \cdot B \cdot L \cdot H+b_{12} \cdot L^{3}+ \\
b_{13} \cdot L \cdot B^{2}+b_{14} \cdot L \cdot H^{2}+b_{15} \cdot L^{2} \cdot B+b_{16} \cdot B^{3}+b_{17} \cdot B \cdot H^{2}+ \\
b_{18} \cdot L^{2} \cdot H+b_{19} \cdot B^{2} \cdot H+b_{20} \cdot H^{3} \\
N u m_{s}(B, L, H)=c_{1}+c_{2} \cdot L+c_{3} \cdot B+c_{4} \cdot H+c_{5} \cdot L \cdot B+c_{6} \cdot L \cdot H+ \\
c_{7} \cdot B \cdot H+c_{8} \cdot L^{2}+c_{9} \cdot B^{2}+c_{10} \cdot H^{2}+c_{11} \cdot B \cdot L \cdot H+c_{12} \cdot L^{3}+ \\
c_{13} \cdot L \cdot B^{2}+c_{14} \cdot L \cdot H^{2}+c_{15} \cdot L^{2} \cdot B+c_{16} \cdot B^{3}+c_{17} \cdot B \cdot H^{2}+ \\
c_{18} \cdot L^{2} \cdot H+c_{19} \cdot B^{2} \cdot H+c_{20} \cdot H^{3} \\
D_{e n}(B, L, H)=d_{1}+d_{2} \cdot L+d_{3} \cdot B+d_{4} \cdot H+d_{5} \cdot L \cdot B+d_{6} \cdot L \cdot H+ \\
d_{7} \cdot B \cdot H+d_{8} \cdot L^{2}+d_{9} \cdot B^{2}+d_{10} \cdot H^{2}+d_{11} \cdot B \cdot L \cdot H+d_{12} \cdot L^{3}+ \\
d_{13} \cdot L \cdot B^{2}+d_{14} \cdot L \cdot H^{2}+d_{15} \cdot L^{2} \cdot B+d_{16} \cdot B^{3}+d_{17} \cdot B \cdot H^{2}+ \\
d_{18} \cdot L^{2} \cdot H+d_{19} \cdot B^{2} \cdot H+d_{20} \cdot H^{3}
\end{array}\right.
$$

where $(l, s)$ are the image point coordinates, $\left(l_{0}, s_{0}\right)$ is the standardized translation parameter of the image point coordinates, and $\left(l_{s}, s_{s}\right)$ is the standardized scale parameter of the image point coordinates. $(B, L, H)$ are the standardized ground point coordinates, $\left(\phi^{\prime}, \lambda^{\prime}, h^{\prime}\right)$ are the ground point space coordinates, $\left(\phi_{0}, \lambda_{0}, h_{0}\right)$ is the normalized translation parameter of the ground point coordinates, and $\left(\phi_{s}, \lambda_{s}, h_{s}\right)$ is the normalized scale parameter of the ground point coordinates. $a_{i}, b_{i}, c_{i}, d_{i}(i=1,2, \cdots, 20)$ are the RPC parameters. The purpose of normalizing the coordinates of the image points $(l, s)$ and the coordinates of the ground points $(\phi, \lambda, h)$ is to avoid rounding errors due to large differences in the magnitude of the data during calculation.

Considering the calibration residuals as the image system bias, the relationship between the image point coordinates and the corresponding ground point coordinates can be expressed as:

$$
\left\{\begin{array}{l}
l+\delta l=\frac{\operatorname{Num}_{L}(B, L, H)}{\operatorname{Den}_{L}(B, L, H)} l_{s}+l_{0} \\
s+\delta s=\frac{\operatorname{Num}_{S}(B, L, H)}{N(B, L, H)} s_{s}+s_{0}
\end{array}\right.
$$

$$
\begin{aligned}
& \delta l=\alpha_{0}+\alpha_{1} l+\alpha_{2} s+\alpha_{3} l^{2}+\alpha_{4} l s+\alpha_{5} s^{2}+\cdots \\
& \delta s=\beta_{0}+\beta_{1} l+\beta_{2} s+\beta_{3} l^{2}+\beta_{4} l s+\beta_{5} s^{2}+\cdots
\end{aligned}
$$

where $\delta l$ and $\delta s$ are the image coordinate biases, and $\alpha_{i}, \beta_{i}(i=1,2,3 \ldots)$ are the image-space bias model parameters. According to the different choices of parameters, the imagespace bias model can be divided into the following models:

(1) Translation bias model

$$
\begin{aligned}
& \delta l=\alpha_{0} \\
& \delta s=\beta_{0}
\end{aligned}
$$

(2) Translation + Drift bias model

$$
\begin{aligned}
& \delta l=\alpha_{0}+\alpha_{1} l \\
& \delta s=\beta_{0}+\beta_{1} l
\end{aligned}
$$

(3) Affine transformation bias model

$$
\begin{aligned}
& \delta l=\alpha_{0}+\alpha_{1} l+\alpha_{2} s \\
& \delta s=\beta_{0}+\beta_{1} l+\beta_{2} s
\end{aligned}
$$

According to the above image-space bias model, combined with the ground control points, the corresponding error equation can be written, and the bias model coefficients can be solved. However, when the affine transformation model cannot simulate satellite on-orbit geometric calibration residuals well, the positioning accuracy of satellite images will still be affected. When solving regional bundle adjustment, the accuracy of the result based on the affine transformation model is highly related to the overlap between the images. Moreover, the larger the distance from the ground control point in the area, the more obvious the magnification effect of the positioning error is (the larger the distance from the control point, the larger the error is) (Pan et al., 2018).

\subsection{Object-Related RPC Bias Model}

In the noncalibration area, the residual performance after satellite calibration is obvious, which is mainly because the thermal and mechanical effects on satellites are not consistent with the calibration. There is a certain correlation between the satellite space thermal characteristics or mechanics and the satellite's operating position. The resulting residual distribution of the calibration also shows a correlation with the satellite's operating position. Therefore, the real geographic coordinates corresponding to the image points can be introduced into the RPC bias model to compensate to better simulate the calibration residuals and further improve the positioning accuracy of satellite images.

The spatial coordinates $(\phi, \lambda, h)$ of the ground point corresponding to the image point is introduced into the RPC bias model to obtain the following polynomial model combining the image-space and object-space models

$$
\begin{aligned}
& \delta l=\alpha_{0}+\alpha_{1} \phi+\alpha_{2} \lambda+\alpha_{3} \phi^{2}+\alpha_{4} \phi \lambda+\alpha_{5} \lambda^{2}+\cdots \\
& \delta s=\beta_{0}+\beta_{1} \phi+\beta_{2} \lambda+\beta_{3} \phi^{2}+\beta_{4} \phi \lambda+\beta_{5} \lambda^{2}+\cdots
\end{aligned}
$$

According to the different parameters, we give two polynomial bias models:

(1) First-order bias model

$$
\begin{aligned}
& \delta l=\alpha_{0}+\alpha_{1} \phi+\alpha_{2} \lambda \\
& \delta s=\beta_{0}+\beta_{1} \phi+\beta_{2} \lambda
\end{aligned}
$$

(2) Third-order bias model

$$
\begin{aligned}
& \delta l=\alpha_{0}+\alpha_{1} \phi+\alpha_{2} \lambda+\alpha_{3} \phi^{2}+\alpha_{4} \phi \lambda+ \\
& \alpha_{5} \lambda^{2}+\alpha_{6} \phi^{2} \lambda+\alpha_{7} \phi \lambda^{2}+\alpha_{8} \phi^{3}+\alpha_{9} \lambda^{3} \\
& \delta s=\beta_{0}+\beta_{1} \phi+\beta_{2} \lambda+\beta_{3} \phi^{2}+\beta_{4} \phi \lambda+ \\
& \beta_{5} \lambda^{2}+\beta_{6} \phi^{2} \lambda+\beta_{7} \phi \lambda^{2}+\beta_{8} \phi^{3}+\beta_{9} \lambda^{3}
\end{aligned}
$$


Because $(\phi, \lambda, h)$ is only for the geographical spatial position distribution corresponding to the image point, it does not require very accurate real coordinates. In actual applications, making $h=0,(\phi, \lambda)$ can be calculated from the image coordinates $(l, s)$ and RPC parameters and then brought into the bias model to calculate the model coefficients. Compared with the traditional model, the essence is to change the correlation of the traditional RPC bias model on the image space to the correlation on the object space. Because the proposed bias model is correlated on the object space, it is also more convenient to unify the multiview images in the area to the object space for processing, increasing the robustness of subsequent overall area adjustment processing.

Taking the first-order bias model as an example, the conversion relationship between the image space and the object space can be written as

$$
\left\{\begin{array}{l}
l+\alpha_{0}+\alpha_{1} \phi+\alpha_{2} \lambda=\frac{\operatorname{Num}_{L}(B, L, H)}{\operatorname{Den}_{L}(B, L, H)} l_{s}+l_{0} \\
s+\beta_{0}+\beta_{1} \phi+\beta_{2} \lambda=\frac{\operatorname{Num}_{S}(B, L, H)}{\operatorname{Den}_{S}(B, L, H)} s_{s}+s_{0}
\end{array}\right.
$$

Therefore,

$$
\left\{\begin{array}{l}
F_{l}(B, L, H)=\frac{\operatorname{Num}_{L}(B, L, H)}{\operatorname{Den}_{L}(B, L, H)} \\
F_{s}(B, L, H)=\frac{\operatorname{Num}_{S}(B, L, H)}{\operatorname{Den}_{S}(B, L, H)}
\end{array}\right.
$$

The error equation can be written as follows:

$$
\left\{\begin{array}{l}
v_{l}=G_{l}\left(\alpha_{0}, \alpha_{1}, \alpha_{2}, B, L, H\right)=F_{l}(B, L, H) l_{s}+l_{0}-\alpha_{0}-\alpha_{1} \phi-\alpha_{2} \lambda \\
v_{s} \lambda G_{s}\left(\beta_{0}, \beta_{1}, \beta_{2}, B, L, H\right)=F_{s}(B, L, H) l_{s}+s_{0}-\beta_{0}-\beta_{\phi} \phi-\beta_{2} \lambda
\end{array}\right.
$$

If the $(B, L, H)$ coordinate error of the ground point coordinates is not considered, the above error equation only includes the bias model coefficients, no linearization is required, and it can be directly solved. If the corresponding ground point coordinate error is considered, the unknown parameters (including the bias model coefficients and the ground point coordinates) can be expanded according to Taylor's theorem and retained to the first-order term

$$
\left\{\begin{array}{l}
v_{l}=G_{l}^{0}+\frac{\partial G_{l}}{\partial \alpha_{0}} d \alpha_{0}+\frac{\partial G_{l}}{\partial \alpha_{1}} d \alpha_{1}+\frac{\partial G_{l}}{\partial \alpha_{2}} d \alpha_{2}+\frac{\partial G_{l}}{\partial B} d B+\frac{\partial G_{l}}{\partial L} d L+\frac{\partial G_{l}}{\partial H} d H \\
v_{s}=G_{s}^{0}+\frac{\partial G_{s}}{\partial \beta_{0}} d \beta_{0}+\frac{\partial G_{s}}{\partial \beta_{1}} d \beta_{1}+\frac{\partial G_{s}}{\partial \beta_{2}} d \beta_{2}+\frac{\partial G_{s}}{\partial B} d B+\frac{\partial G_{s}}{\partial L} d L+\frac{\partial G_{s}}{\partial H} d H
\end{array}\right.
$$

Thus, the corresponding bias model coefficients and ground point coordinates can be solved iteratively. In the case of considering the $(B, L, H)$ coordinate error of the ground point coordinates, since the $(\phi, \lambda, h)$ term introduced in the bias model is only for the geographical space position distribution corresponding to the image point. To increase the robustness of the solution process, $(\phi, \lambda, h)$ can still continue to keep the initial value unchanged and just modifying the value of $(B, L, H)$.

\section{EXPERIMENT AND ANALYSIS}

\subsection{Experimental Data}

Experimental verification was performed using TH-1 satellite image data. The TH-1 satellite is the first Chinese stereo mapping satellite. It is equipped with a 5-meter-resolution three-

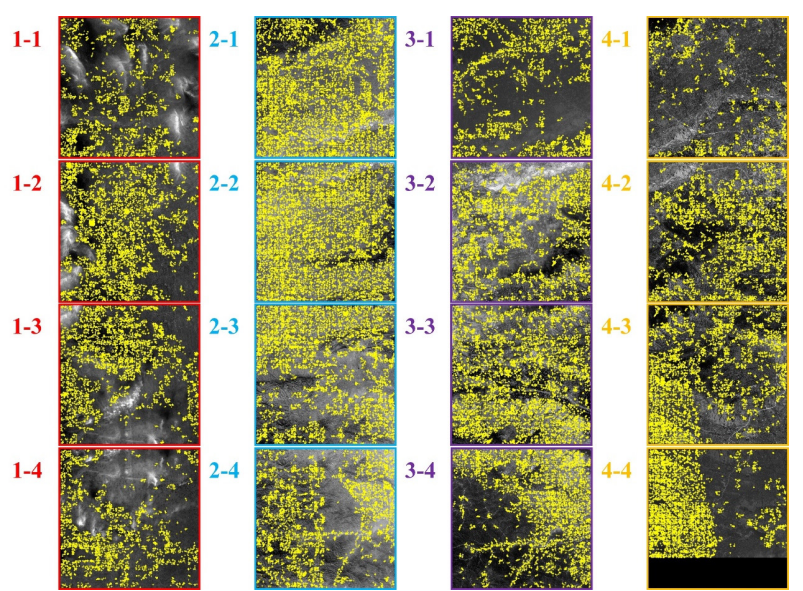

Figure 1 Distributions of the image and ground control points -line-array CCD camera, a 2-meter-resolution full-color camera, and a 10-meter-resolution multispectral camera. Three-line imagery can be used for topographic mapping at the 1:50000 scale. The experiments used four groups of three-line array nadir-viewing images taken in November 2010, November 2012, April 2013, and May 2014. Each dataset contains 4 scenes of the same-track image and corresponding RPC data. The 2-meter-resolution aerial orthophoto of the corresponding area as the reference data and use the AW3D30 data to obtain the corresponding ground point elevation data. A variety of matching algorithms were used to automatically match satellite and aerial DOM imagery data to obtain a total of 171,106 ground control points, the distribution of which is shown in Figure 1.

The background image in Figure 1 is the original satellite image. From left to right: the first dataset (2010); the second dataset (2012); the third dataset (2013); the fourth dataset (2014). The yellow dots indicate the distribution of the ground control points extracted automatically. The numbers on the left side of the image are the image numbers. The upper and lower overlap between the same-track images in each dataset is $20 \%$. Among them, image 4-4 is nonstandard scene data, and there are no data for approximately 2,000 lines, but these missing data not affect the RPC accuracy evaluation experiment. As seen from Figure 1, except for the cloud occlusion area in the image, the number of ground control points is sufficient, and the distribution is relatively uniform, which can meet the requirements of RPC correction and accuracy evaluation.

\subsection{Experimental Results and Analysis}

The original RPC of the image is used to backproject the coordinates of the ground control point to the image space, and the plane distance is calculated from the image coordinates of the ground control point. Then, the original RPC error image plane accuracy distribution of the image space can be obtained. The resulting original RPC accuracy distribution is shown in Figure 2. 

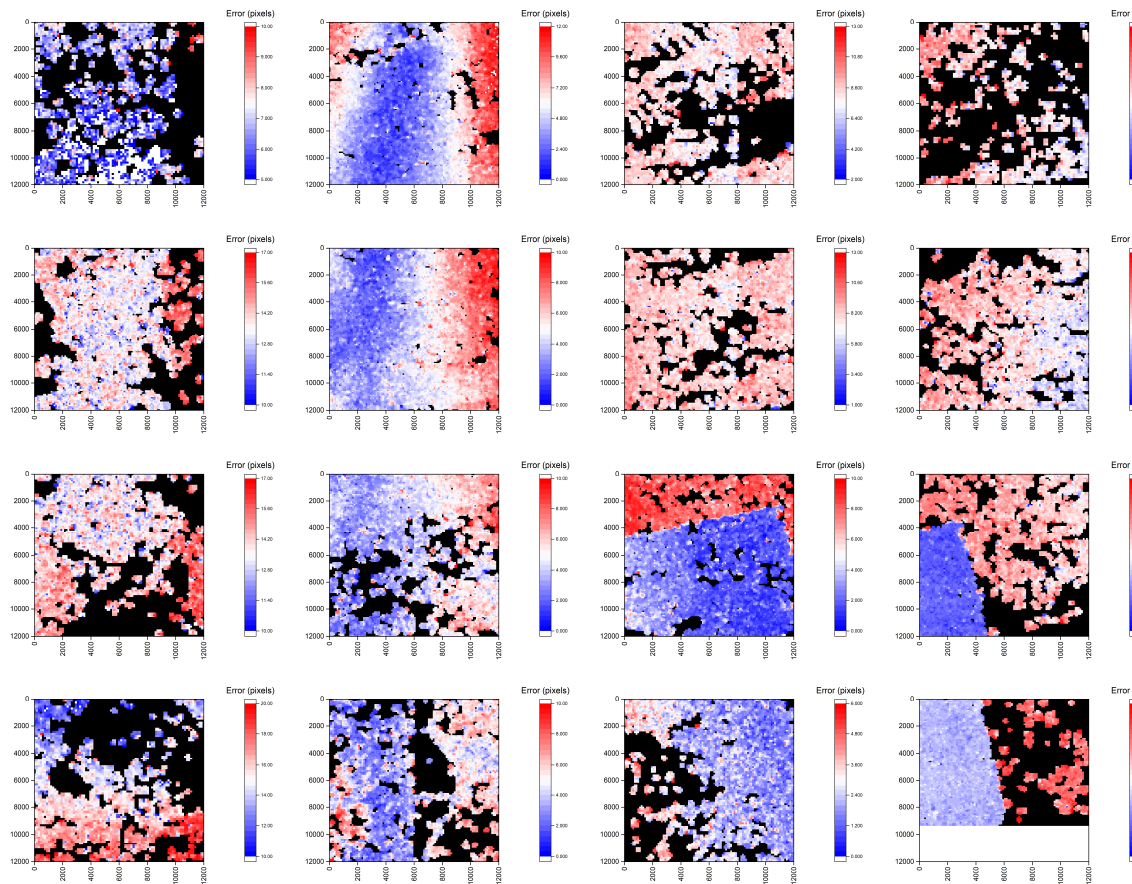

Figure 2 Original error distribution

Table 1 Statistics of the single-model correction accuracy (unit: pixels)

\begin{tabular}{|c|c|c|c|c|c|c|c|c|c|c|}
\hline \multirow{2}{*}{$\begin{array}{l}\text { Image } \\
\text { number }\end{array}$} & \multicolumn{2}{|c|}{ Raw accuracy } & \multicolumn{2}{|c|}{ Translation model } & \multicolumn{2}{|c|}{$\begin{array}{l}\text { Affine transformation } \\
\text { model }\end{array}$} & \multicolumn{2}{|c|}{ First-order model (ours) } & \multicolumn{2}{|c|}{ Third-order model (ours) } \\
\hline & RMSE & CE90 & RMSE & CE90 & RMSE & CE90 & RMSE & CE90 & RMSE & CE90 \\
\hline $1-1$ & 6.08 & 8.33 & 1.84 & 3.86 & 1.87 & 3.87 & 1.75 & 3.79 & 1.74 & 3.78 \\
\hline $1-2$ & 13.72 & 15.84 & 1.68 & 3.49 & 1.70 & 3.51 & 1.67 & 3.50 & 1.67 & 3.49 \\
\hline $1-3$ & 14.04 & 16.09 & 1.93 & 3.72 & 1.92 & 3.73 & 1.86 & 3.69 & 1.85 & 3.69 \\
\hline $1-4$ & 15.38 & 18.27 & 2.95 & 5.04 & 2.92 & 5.02 & 2.51 & 4.51 & 2.50 & 4.53 \\
\hline $2-1$ & 4.89 & 9.60 & 4.62 & 8.94 & 4.63 & 8.82 & 4.63 & 8.78 & 4.60 & 8.74 \\
\hline $2-2$ & 4.26 & 7.81 & 3.87 & 7.82 & 3.87 & 7.78 & 3.90 & 7.70 & 3.83 & 7.59 \\
\hline $2-3$ & 4.26 & 7.08 & 4.12 & 6.63 & 2.97 & 6.36 & 2.84 & 5.94 & 2.77 & 5.75 \\
\hline $2-4$ & 4.16 & 7.20 & 3.82 & 7.10 & 2.71 & 5.26 & 2.68 & 5.25 & 2.60 & 5.12 \\
\hline $3-1$ & 8.20 & 10.28 & 5.86 & 7.77 & 5.86 & 7.83 & 5.86 & 7.77 & 5.86 & 7.77 \\
\hline $3-2$ & 8.20 & 10.22 & 5.44 & 7.33 & 5.44 & 7.36 & 5.43 & 7.33 & 5.43 & 7.33 \\
\hline $3-3$ & 2.62 & 8.32 & 2.77 & 6.87 & 2.46 & 6.67 & 2.56 & 5.82 & 2.40 & 5.81 \\
\hline $3-4$ & 2.03 & 4.15 & 1.84 & 3.88 & 1.78 & 3.71 & 1.73 & 3.69 & 1.74 & 3.69 \\
\hline $4-1$ & 5.74 & 8.10 & 1.95 & 4.14 & 1.85 & 4.05 & 1.83 & 4.02 & 1.83 & 4.01 \\
\hline $4-2$ & 10.68 & 12.77 & 9.25 & 11.22 & 9.20 & 11.22 & 9.21 & 11.19 & 9.22 & 11.19 \\
\hline $4-3$ & 9.10 & 12.04 & 8.08 & 10.79 & 7.89 & 10.99 & 7.88 & 10.71 & 7.84 & 10.67 \\
\hline $4-4$ & 4.85 & 10.14 & 4.92 & 9.86 & 5.00 & 9.20 & 4.98 & 9.20 & 4.98 & 9.20 \\
\hline
\end{tabular}

Figure 2 shows that the error distribution in each scene is different, and the traditional RPC bias model cannot adapt well to the residual situation.

The two object-related RPC bias models proposed in this paper are compared with the traditional translation model and affine transformation model, producing four comparison methods for the experiments. For the four satellite image datasets, adjustments based on ground control points are performed for each image to evaluate the effectiveness of the bias model. The experimental results are shown in Table 1.

Table 1 uses two indexes, RMSE and CE90, to quantify the positioning accuracy. The values in the green columns are the RMSE errors of each image, the values in the blue columns are the CE90 errors of each image, the purple values are the minimum RMSE values after adjustment, and the red values are the minimum CE90 values after adjustment. 
Table 2 Statistics of the correction accuracy of shared model (unit: pixels)

\begin{tabular}{|c|c|c|c|c|c|c|c|c|c|c|}
\hline \multirow{2}{*}{ Image number } & \multicolumn{2}{|c|}{ Raw accuracy } & \multicolumn{2}{|c|}{ Translation model } & \multicolumn{2}{|c|}{$\begin{array}{c}\text { Affine transformation } \\
\text { model }\end{array}$} & \multicolumn{2}{|c|}{ First-order model (ours) } & \multicolumn{2}{|c|}{$\begin{array}{c}\begin{array}{c}\text { Third-order model } \\
\text { (ours) }\end{array} \\
\end{array}$} \\
\hline & RMSE & CE90 & RMSE & CE90 & RMSE & CE90 & RMSE & CE90 & RMSE & CE90 \\
\hline $1-1$ & 6.08 & 8.33 & 6.79 & 9.15 & 6.81 & 9.15 & 3.99 & 6.68 & 3.84 & 6.74 \\
\hline $1-2$ & 13.72 & 15.84 & 2.04 & 3.93 & 2.05 & 3.95 & 2.83 & 5.05 & 2.47 & 4.70 \\
\hline $1-3$ & 14.04 & 16.09 & 2.39 & 4.23 & 2.37 & 4.21 & 1.95 & 3.80 & 1.83 & 3.70 \\
\hline $1-4$ & 15.38 & 18.27 & 3.80 & 6.46 & 3.80 & 6.47 & 2.76 & 4.88 & 2.76 & 4.84 \\
\hline Average value & 12.31 & 14.63 & 3.75 & 5.94 & 3.76 & 5.94 & 2.88 & 5.10 & 2.73 & 4.99 \\
\hline $2-1$ & 4.89 & 9.60 & 4.78 & 9.45 & 4.83 & 10.11 & 4.79 & 9.46 & 4.72 & 8.98 \\
\hline $2-2$ & 4.26 & 7.81 & 4.12 & 7.78 & 4.00 & 7.96 & 3.99 & 7.78 & 3.88 & 7.71 \\
\hline $2-3$ & 4.26 & 7.08 & 4.34 & 7.26 & 3.96 & 6.71 & 3.42 & 6.28 & 3.06 & 6.01 \\
\hline $2-4$ & 4.16 & 7.20 & 4.25 & 7.32 & 3.68 & 6.41 & 3.15 & 6.30 & 2.76 & 5.40 \\
\hline Average value & 4.39 & 7.92 & 4.37 & 7.95 & 4.12 & 7.80 & 3.84 & 7.45 & 3.61 & 7.03 \\
\hline $3-1$ & 8.20 & 10.28 & 6.23 & 8.19 & 6.25 & 8.31 & 5.98 & 7.92 & 5.92 & 7.86 \\
\hline $3-2$ & 8.20 & 10.22 & 6.00 & 7.89 & 5.98 & 7.96 & 5.71 & 7.62 & 5.61 & 7.49 \\
\hline $3-3$ & 2.62 & 8.32 & 3.81 & 6.29 & 3.65 & 6.20 & 2.89 & 6.05 & 2.69 & 5.88 \\
\hline $3-4$ & 2.03 & 4.15 & 3.18 & 5.20 & 2.94 & 4.99 & 1.93 & 3.87 & 1.82 & 3.78 \\
\hline Average value & 5.26 & 8.24 & 4.80 & 6.90 & 4.71 & 6.87 & 4.13 & 6.36 & 4.01 & 6.25 \\
\hline $4-1$ & 5.74 & 8.10 & 2.73 & 5.02 & 2.90 & 5.62 & 2.27 & 4.69 & 1.87 & 4.08 \\
\hline $4-2$ & 10.68 & 12.77 & 9.43 & 11.42 & 9.49 & 11.57 & 9.37 & 11.33 & 9.26 & 11.22 \\
\hline $4-3$ & 9.10 & 12.04 & 8.05 & 10.71 & 7.90 & 10.85 & 7.96 & 10.78 & 7.91 & 10.66 \\
\hline $4-4$ & 4.85 & 10.14 & 6.15 & 9.26 & 5.74 & 9.15 & 5.04 & 9.41 & 5.04 & 9.17 \\
\hline Average value & 7.59 & 10.76 & 6.59 & 9.10 & 6.51 & 9.30 & 6.16 & 9.05 & 6.02 & 8.78 \\
\hline
\end{tabular}

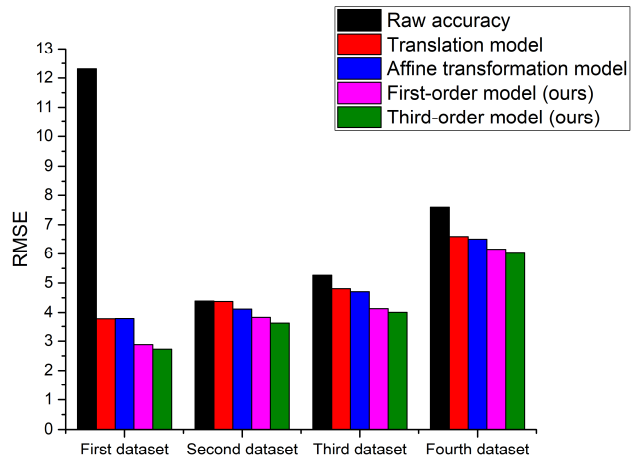

(a) RMSE

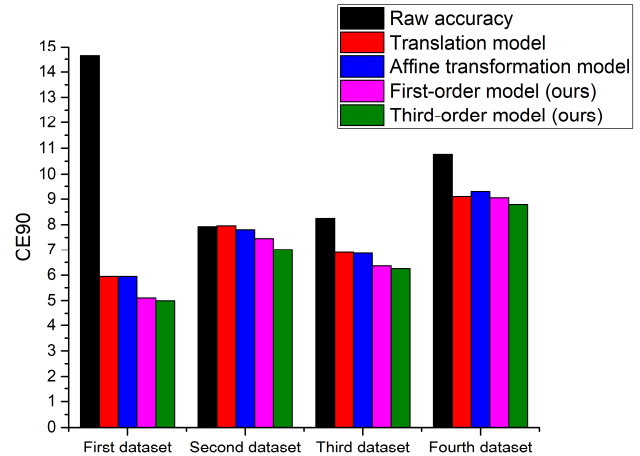

(b) CE90

Figure 3 Adjustment accuracy statistics

It can be seen that in the adjustment results for each image, the bias model proposed in this paper achieved better correction results in terms of both the RMSE and CE90. Compared with the traditional translation bias model, the third-order bias model proposed in this paper can improve the accuracy by 1.35 pixels on the RMSE and 1.98 pixels on the CE90. Compared with the original accuracy, the proposed model can improve the accuracy by up to 12.88 pixels in terms of the RMSE by up to 13.74 pixels in terms of the CE90. As seen from Table 1, the RPC bias model proposed in this paper can effectively eliminate some errors. Overall, it can achieve the best results, which illustrates the effectiveness of the bias model in this paper.
For the four groups of satellite image data, the images in each group share the same bias model parameters for the same orbit image to evaluate the overall stability of the correction model. Adjustment is carried out based on the ground control points. The final experimental results are shown in Table 2 and Figure 3. Table 2 uses two indexes, RMSE and CE90, to quantify the positioning accuracy. The values in the green columns are the RMSE errors of each image, the values in the blue columns are the CE90 errors of each image, the orange number is the average accuracy for each dataset, the purple value is the minimum RMSE value after adjustment for each dataset, and the red value is the minimum CE90 value after adjustment for 
each dataset. In Figure 3, (a) is an average statistical histogram of the RMSE errors for each dataset, and (b) is an average statistical histogram of the CE90 errors for each dataset. It can be seen from Table 2 and Figure 3 that the model proposed in this paper uses the shared bias model parameters for adjustment on the same orbit image, and the final accuracy achieved is the best for the experimental data. Compared with the traditional translation and affine transformation model, the plane accuracy can be improved by approximately 1 pixel at most, which shows that the model proposed in this paper has strong robustness. This property is very important for RPC adjustment applications with sparse control points.

\section{CONCLUSION}

To optimize the positioning accuracy of satellite images, a new RPC bias model is proposed in this paper. The feasibility of this method is verified by experiments on multiple satellite image datasets and a comparative analysis with the traditional RPC bias model. The experimental results show that the proposed method can effectively improve the plane accuracy by approximately 1 pixel compared with the traditional translation bias model. In the next step, we will use a variety of on-orbit satellite image data for adjustment experiments to further verify the applicability of this method.

\section{ACKNOWLEDGEMENTS}

This work was supported in part by the National Natural Science Foundation of China under Grant 41401534 and Grant 41971427.

\section{REFERENCES}

Cao, H., Tao, P., Li, H., Shi, J., 2019. Bundle adjustment of satellite images based on an equivalent geometric sensor model with digital elevation model. ISPRS Journal of Photogrammetry and Remote Sensing 156, 169-183.

Dong, Y., Fan, D., Ma, Q., Ji, S., Ouyang, H., 2019. Automatic on-orbit geometric calibration framework for geostationary optical satellite imagery using open access data. International Journal of Remote Sensing 40 (16), 6154-6184.

Fraser, C.S., Dial, G., Grodecki, J., 2006. Sensor orientation via RPCs. ISPRS Journal of Photogrammetry and Remote Sensing 60, 182-194.

Fraser, C.S., Hanley, H.B., 2003. Bias compensation in rational functions for IKONOS satellite imagery. Photogrammetric Engineering and Remote Sensing 69 (1), 53-57.

Fraser, C.S., Hanley, H.B., 2005. Bias-compensated RPCs for sensor orientation of high-resolution satellite imagery. Photogrammetric Engineering and Remote Sensing 71 (8), 909915.

Grodecki, J., Dial, G., 2003. Block adjustment of highresolution satellite images described by rational rolynomials. Photogrammetric Engineering and Remote Sensing 69 (1), 5968.

Jiang, Y., Cui, Z., Zhang, G., Wang, J., Xu, M., Zhao, Y., Xu, Y., 2018. CCD distortion calibration without accurate ground control data for pushbroom satellites. ISPRS Journal of Photogrammetry and Remote Sensing 142, 21-26.
Li D., 2019. Towards geospatial information technology in 5G/6G era. Acta Geodaetica et Cartographica Sinica 48 (12), 1475-1481.

Noh, M., Howat, I. M., 2018. Automatic relative RPC image compensation through hierarchical image matching for improving DEM quality. ISPRS Journal of Photogrammetry and Remote Sensing 136, 120-133.

Pan, H., Zou, S., Guan, L., 2018. Block Adjustment with Drift Compensation for Rational Function Model. Photogrammetric Engineering and Remote Sensing 84 (12), 791-799.

Tong, X., Liu, S., Weng, Q., 2010. Bias-corrected rational polynomial coefficients for high accuracy geo-positioning of QuickBird stereo imagery. ISPRS Journal of Photogrammetry and Remote Sensing 65, 218-226.

Wang, J., Wang, R., Hu, X., Su, Z., 2017. The on-orbit calibration of geometric parameters of the Tian-Hui 1 (TH-1) satellite. ISPRS Journal of Photogrammetry and Remote Sensing 124, 144-151.

Wang, M., Cheng, Y., Chang, X., Jin, S., Zhu, Y., 2017. Onorbit geometric calibration and geometric quality assessment for the high-resolution geostationary optical satellite GaoFen4. ISPRS Journal of Photogrammetry and Remote Sensing 125, 63-77.

Yang, B., 2016. Accuracy Validation of Large-scale Block Adjustment without Control of ZY3 Images over China. ISPRS Annals of the Photogrammetry, Remote Sensing and Spatial Information Sciences, 105-112.

Yang, B., Wang, M., Xu, W., Li, D., Pi, Y., 2017. Large-scale block adjustment without use of ground control points based on the compensation of geometric calibration for zy-3 images. ISPRS Journal of Photogrammetry and Remote Sensing 134, 114.

Zhang, L., Zhang, J., Chen, X., An, H., 2009. Block-adjustment with SPOT-5 imagery and sparse GCPs based on RFM. Acta Geodaetica et Cartographica Sinica 38 (4), 302-310. 